and hip fracture between those calculated with BMD and those without BMD measurements $(P>0.05)$ respectively among female patients $(n=32)$.

Conclusions: A substantial gap exists between FRAX with BMD and without BMD in Korean patients with AS.

Disclosure of Interest: None declared

DOI: 10.1136/annrheumdis-2017-eular.5575

\section{AB0856 T-SCORE OF THE SPINE AS PREDICTOR OF THE FEMORAL NECK FRACTURE}

S.D. Jandric. Department for FRM, Faculty of Medicine, University of Banjaluka, Banja Luka, Republic of Srpska, Bosnia and Herzegovina

Background: Osteoporosis is defined as a progressive, systemic skeletal disorder characterized by low bone mass and micro-architectural deterioration of bone tissue with a consequent increase in bone fragility and susceptibility to fracture. There are numerous hip fracture risks. Bone mineral density (BMD) and T-score measured by dual-energy X-ray absorptiometry (DXA) are the main determinants of the clinical evaluation of hip fracture risk. World Health Organization classification defined osteoporosis as T-score below -2.5 SD.

Objectives: The aim of this study was to estimate differences in DXA measurements (BMD and T-score of the spine) and potential predictors of the femoral neck fracture in the patients with osteoporosis.

Methods: This study included 181 patients with osteoporosis (165 female and 16 male), average age of the $65,6 \pm 8.5$ years (range of 44.1 to 87.3 years). Eighty one patients had fracture of the femoral neck. All patients in this group were managed operatively by hip arthroplasty, after clinical and radiological diagnostic procedures. DXA measurement was performed on Advanced Prodigy Lunar device for these patients postoperatively. BMD of the femoral neck was measured on the no operated side. Age, sex, height, weight, BMI, BMD and T-score of the spine at the level of L1-L4, BMD of the right and left femoral neck were estimated. The control group included 100 patients with osteoporosis (93 female and 7 male), average age of the $65.1 \pm 8,5$ years. Student's t-test and Logistic regression were used for statistical analysis. Dependent variable was presence of the fracture of the femoral neck and independent variables were age, sex, height, weight, BMI, BMD and T-score of the spine and BMD of the femoral neck.

Results: Results of our study showed statistically significant difference between T-score of the spine $(t=-2,973, p<0.01)$ as well as between BMD of the spine $(t=-12,376, p<0.001)$ of patients with and without fracture of the femoral neck. T-score of the spine was significant predictor of fracture of the femoral neck $(p<0$, 01) when controlled by age, sex, height, weight, BMI, BMD of the spine, BMD of the femoral neck.

Conclusions: T-score and BMD of the spine were statistically significantly lower in patients with fracture of the femoral neck than in patients with osteoporosis without fracture. T-score of the lumbar spine was significant predictor of fracture of the femoral neck in patients with osteoporosis. Probability of femur neck fractures increased with the decrease of T-score of lumbar spine in patients with osteoporosis. These results can help in predicting femur neck fractures.

References:

[1] British National Formulary. September 2005; 385-9.

[2] Vid KS JA, Thien A, Hill R. Ann R. Implementation of and compliance with NICE guidelines in the secondary prevention of osteoporotic fractures in postmenopausal women. Coll Surg Engl 2008; 90: 213-5.

[3] Del Rio LM, et al. Is bone microarchitecture status of the lumbar spine assessed by TBS related to femoral neck fracture? A Spanish case-control study. Osteoporos Int. 2013; 24(3):991-8.

Disclosure of Interest: None declared

DOI: 10.1136/annrheumdis-2017-eular.2768

\section{AB0857 PROBLEMS OF DIAGNOSTICS AND PROPHYLAXIS OF GLUCOCORTICOID-INDUCED OSTEOPOROSIS IN REAL CLINICAL PRACTICE}

S. Yakupova, R. Demidov. Kazan State Medical University, Kazan, Russian Federation

Background: Oral glucocorticoids (GC) are used in different medicine fields and appear as risk factors of glucocorticoid-induced osteoporosis (GIO).

Objectives: The aim is to estimate the frequency of use of prophylaxis of OP, use drugs that are approved for $\mathrm{GIO}$ and also an awareness of $\mathrm{GIO}$ of patients with prolonged intake of GC.

Methods: 50 patients ( 10 men and 40 women), taking GC, took part in research. 30 patients $(60 \%)$ were from rheumatology department, 5 patients $(10 \%)$ from pulmonology department, 5 patients $(10 \%)$ from gastroenterology department, 10 patients $(20 \%)$ from nephrology department of Republic Clinical Hospital. Mean age of patients - 48,84 $\pm 14,03$ years (from 26 to 73 ). The following signs were estimated: clinical data, osteoporosis risk factors, instrumental tests (X-ray, densitometry). FRAX assessment of fracture risk was performed, the questionnaires of patients' awareness of GIO was completed.

Results: The duration of intake GC $-5,93 \pm 4,86$ years. Minimal dose of GC per day (if receiving prednisone) $-7.5 \mathrm{mg}$; maximal dose $-60 \mathrm{mg}$. 10-year risk of major osteoporotic fractures by FRAX, adjusted according to GC dose

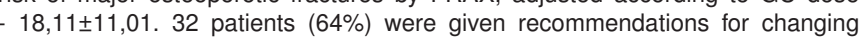

lifestyle and diet for GIO prophylaxis, 40 patients (80\%) - recommendations for intake of calcium and vitamin D medications, but only 31 patients $(62 \%)$ followed recommendations and started the intake of calcium medications. From the said number of patients only 14 patients $(45,2 \%)$ used appropriate daily dose of calcium and vitamin D.

Drugs that are approved for GIO were have to be prescribed for 18 patients, but only 6 patients $(33,3 \%)$ underwent treatment, principally bisphosphonates. Only half of them underwent densitometry after starting the therapy. $72,2 \%$ patients with GIO used calcium and $30,7 \%$ were taking appropriate daily dose of calcium and vitamin D. $70 \%$ rheumatologic patients knew about GIO and in $90 \%$ cases calcium and vitamin D drugs were recommended. Only $50 \%$ of patients from non-rheumatologic departments knew about GIO and in $65 \%$ cases calcium and vitamin $\mathrm{D}$ drugs were recommended.

Conclusions: Clinical recommendations in real clinical practice are rarely fulfilled. Less than $60 \%$ patients were taking calcium and vitamin D, which are recommended for all patients, who started the GC therapy; only $33 \%$ of patients received osteoporosis therapy, only half of them underwent densitometry. Patients are insufficiently informed about necessity of changing lifestyle and diet for GIO prophylaxis. Education for patients taking GC and training for rheumatologic and non-rheumatologic specialties are necessary.

Disclosure of Interest: None declared

DOI: 10.1136/annrheumdis-2017-eular.6867

\section{AB0858 ASSOCIATION OF BONE MINERAL DENSITY WITH DEVELOPMENT OF HEART FAILURE IN DIABETIC PATIENTS}

V. Vasilkova ${ }^{1}$, T. Mokhort ${ }^{2}$, E. Naumenko ${ }^{3}$, M. Zmailik ${ }^{4} .{ }^{1}$ Endocrinology, Gomel State Medical University, Gomel; ${ }^{2}$ Endocrinology, Belarusian State Medical University, Minsk; ${ }^{3}$ Functional Diagnostics; ${ }^{4}$ Endocrinology, The Republican Research Center for Radiation Medicine and Human Ecology, Gomel, Belarus

Background: Diabetes mellitus has shown to be a significant risk factor for the development and prognosis of heart failure (HF) and associated with an increased risk of fractures [1]. Osteoporosis and heart failure are generally considered two distinct diseases, but recent evidence suggests a link between both diseases. Objectives: The aim of the study was to investigate the association of bone mineral density with the risk of developing heart failure in diabetic patients.

Methods: 85 patients both sexes with type 2 diabetes aged $58.69 \pm 9.07$ years were studied. Besides standard laboratory parameters, the echocardiographic and BMD measurements were performed. Estimated glomerular filtration rate was measured.

Results: Among diabetic subjects, 8 patients (9.4\%) had osteoporosis, 21 (24.8\%) had osteopenia and $56(65.8 \%)$ had a normal BMD. Increased serum NT-proBNP $(p<0.001)$ and decreased left ventricular ejection fraction (EF) $(p=0.03)$ were significantly correlated with low T-score L1-L4 cutoff points between groups (normal, osteopenia, and osteoporosis). Multivariate stepwise linear regression analysis of the significant variables revealed that NT-proBNP, EF were independent predictors of lumbar BMD among female patients with diabetes mellitus. After adjusting for age, gender, and related comorbidities, the osteoporosis group was associated with a significantly higher risk of coronary artery disease in women with diabetes. However, no association between BMD and HF was found in men. Conclusions: Osteoporosis may be an independent factor for HF in women with diabetes mellitus. Our data suggested that early detection of abnormal BMD should warrant for early search of undetected HF in diabetic women. A further study is needed to elucidate the effects of BMD on cardiac function in diabetic patients.

\section{References:}

[1] Heart failure is a risk factor for orthopedic fracture: a population-based analysis of 16,294 patients. van Diepen S, Majumdar SR, Bakal JA, McAlister FA, Ezekowitz JA Circulation. 2008 Nov 4; 118(19):1946-52.

Acknowledgements: We acknowledged the help from the Republican Research Center for Radiation Medicine and Human Ecology for the technical assistance. Disclosure of Interest: None declared

DOI: 10.1136/annrheumdis-2017-eular.3867

\section{AB0859 CAN ZOLENDRONIC ACID USE LEAD TO IMPAIR RENAL FUNCTION IN OSTEOPOROSIS PATIENTS?}

Y.-C. Chen, C.-H. Ko. Rheumatology, Kaohsiung Chang Gung Memorial Hospital, Kaohsiung, Taiwan, Province of China

Background: Bisphosphonates are recommended in patients with osteoporosis patients. clinical concerns had been considered in kidney safety.

Objectives: This study investigated the safety of bisphosphonate effects on renal function in patients with magnetic resonance imaging (MRI)-proven acute osteoporotic vertebral fractures after vertebroplasty.

Methods: This retrospective study was conducted in osteoporotic patients with acute vertebral fractures treated with vertebroplasty between January 2001 and December 2015. Their gender, age, body mass index (BMl, $\left.\mathrm{kg} / \mathrm{m}^{2}\right)$, co-morbidities were recorded, as well as their use of zolendronic acid. Those with increase in creatinine was defined as progress of renal function. Logistical regression was used to adjust the variables.

Results: There were 989 patients (783 females; mean age, 74.08 \pm 9.26 years). 